\title{
Strafrechtlicher Umgang mit jungen Männern aus dem Zuwanderungsmilieu - die Rolle des Ausländerrechts \\ Monika Frommel
}

\section{Offene Fragen}

D ie in diesem Heft abgedruckten Titelbeiträge bestätigen nicht die üblichen Erwartungen, dass junge Ausländer schwerere Delikte begehen, häufiger rückfällig seien und deshalb härter sanktioniert werden. Sie belegen vielmehr geradezu erwartungswidrig, dass sich sog. Deutsche und Nichtdeutsche im Dunkelfeld in ihrem Verhalten nicht so sehr unterscheiden, wie wir das bislang angenommen haben, zumindest dann nicht, wenn man die Indikatoren für die Schichtzugehörigkeit konstant hält und bedenkt, dass es im Migrantenmilieu sehr viel mehr junge Männer gibt als in der überalterten deutschen Wohnbevölkerung und dass junge Männer in allen Kulturen eine insgesamt höhere Auffälligkeit aufweisen.

Auch Studien, welche die Rückfälligkeit untersuchen (hierzu III.), kommen zum erwartungswidrigen Ergebnis, dass diese weder generell erhöht sei noch bei den besonders schweren Delikten wie Raub, Vergewaltigung und Körperverletzung. Aber auch hier wird angemerkt, dass die Tatsache, dass Nichtdeutsche unter bestimmten Voraussetzungen abgeschoben werden können, verzerrend wirkt auf die Berechnungen nach den Daten des Bundeszentralregisters. Denn nach $\S \S 53,54$ AufenthaltsG ist die Abschiebung zwingend nach einer Verurteilung zu mehr als drei Jahren geboten und sie ist bei allen Drogendelikten der Regelfall. Ferner ist sie möglich nach einer Verurteilung von zwei Jahren ohne Bewährung. Sie droht also insbesondere bei schwereren Gewaltdelikten. Hinzu kommt noch, dass bei der Strafzu- messung nicht zugunsten des Angeklagten berücksichtigt werden darf, dass ihm nach Versagung der Bewährung die Abschiebung droht. Das Argument ist das, dass die Ausländerbehörde ja noch ein Ermessen habe. So gesehen liegt es schon nahe, dass Angeklagte, die abgeschoben werden können, eher zu einer Freiheitsstrafe ohne Bewährung verurteilt werden.

Nichtdeutsche Angeklagte müssen also beides fürchten. Eine eher negative Prognose, die dazu führt, dass sie häufiger keine Geldstrafe, sondern eine Freiheitsstrafe erhalten. Dass sie dann auch häufiger keine Bewährungsstrafe mehr erhalten als deutsche Angeklagte, und dass damit ihr Risiko erheblich erhöht ist abgeschoben zu werden. Deutsche und Nichtdeutsche können daher auch nur bedingt auf ihre Rückfälligkeit hin verglichen werden, da die Abschiebung nicht erst beim Rückfall, sondern bereits dann erfolgt, wenn eine Freiheitsstrafe von mindestens zwei Jahren abzusitzen ist.

Die hier vertretene These lautet daher. Zwar sind Unterschiede im Hellfeld unbestritten. Es gibt Delikte, die häufiger bei Ausländern registriert werden als bei Deutschen, aber aus diesen Daten lässt sich nicht ohne weiteres auf das Verhalten rück schließen. Die Unterschiede seien aber genannt. Eigentums- und Vermögensdelikte sind bei allen männlichen Beschuldigten etwa gleich verteilt. Auch gleichen sich die Daten der polizeilichen Belastungsziffern bei der einfachen Körperverletzung an. Aber bei der sexuellen Nötigung, den Raubdelikten und der Drogenkriminalität, ferner der gefährlichen Körperverletzung gibt es einen deutlich erhöhten Anteil der sog. Nichtdeutschen: alles Delikte, die tendenziell eher mit einer Freiheitsstrafe geahndet werden. Umgekehrt ist die Rate der nichtdeutschen Beschuldigten bei den Tötungsdelikten und auch sonst eher niedriger. Entscheidend ist aber nicht die Struktur der sog. Ausländerkriminalität im Hellfeld, sondern vor allem die Art und Weise der staatlichen Reaktion auf dieselbe Straffälligkeit. Wer Unterschiede beschreiben will, benötigt eine komplexe Etikettierungstheorie.

Im folgenden wird diese Theorie nicht geliefert. Der Beitrag versteht sich als Anregung, nicht als Antwort auf drängende Fragen.

Gründe für die überproportionale Inhaftierung von Minoritäten werden von Kriminologen so gut wie nie im Ausländerrecht und dessen direkten oder indirekten Auswirkungen gesucht, sondern in den Faktoren, die bei der Erklärung von Kriminalität und Kriminalisierung üblicherweise herangezogen werden, nämlich

- in ihrem unterschiedlichen (ggf. auch strafbaren) Verhalten und ihrer Lebenssituation,

- in der unterschiedlichen tatsächlichen Behandlung durch die Gesellschaft und ihre Kontrollinstanzen einschließlich der Berichterstattung der Massenmedien und

- der unterschiedlichen Behandlung durch das Recht.

Fügen wir eine weitere Hypothese hinzu. Es könnte sein, dass neben den spezifischen auslän- derrechtlichen Sanktionen noch ein weiterer Faktor zu wenig berücksichtigt wird, nämlich die erleichterte und praktisch auch häufig praktizierte Untersuchungshaft wegen Fluchtgefahr - selbst bei eher leichteren Delikten - und die damit veränderte Lage des Beschuldigten und seiner Verteidigung in einem Strafverfahren. Wer schon in Untersuchungshaft sitzt, muss fürchten, dass nun der ausländerrechtliche Status geprüft wird und am Ende auch noch die Abschiebung droht. Sollten beide Faktoren eine dominante Rolle spielen, dann wäre die erhöhte Gefangenenrate eine logische Folge und Konsequenz des Ausländerrechts. Einer der wesentlichen Faktoren müsste in der unterschiedlichen Behandlung durch das Recht gesehen werden. Die hohen Gefangenenraten wären eine gezielte und daneben noch eine unintendierte Folge der vielfältigen Eingriffsbefugnisse des Ausländerrechts, welche in der Kombination mit strafprozessualen Eingriffsbefugnissen besonders hart wirken.

Kehren wir zurück zum Beschuldigten ohne gesicherten ausländerrechtlichen Status und nehmen wir außerdem an, er habe gegen das BtMG verstoßen bzw. eine gefährliche Körperverletzung begangen und/oder sich bei einem Raub und/oder einer sexuellen Nötigung beteiligt.

Die Wahrscheinlichkeit, dass gegen ihn ein Untersuchungshaftbefehl wegen Fluchtgefahr erlassen wird, ist sehr viel höher als bei einem gut integrierten jungen Mann mit deutschem Pass. Aber selbst wenn es gelingen sollte, den Untersuchungshaftbefehl erfolgreich anzugreifen oder zu- 
mindest die Untersuchungshaft außer Vollzug zu setzen, nimmt das ausländerrechtliche Überprüfungsverfahren seinen Lauf und häufig wird dann in der letzten Seite der Akte ein Sicherungshaftbefehl abgeheftet sein, der die Abschiebung vollziehbar macht.

Bei vielen der sog. Nichtdeutschen sind also scharfe ausländerrechtliche Zugriffsmöglichkeiten gegeben wie etwa folgende Kette zeigt: Prüfung des Aufenthaltsrechts nach einer Straftat, Versagen dieses Rechts, Androhung der Abschiebung und schließlich Erlass eines Sicherungshaftbefehls, wenn nicht davon ausgegangen werden kann, dass der in Untersuchungshaft befindliche Beschuldigte nach Rechtskraft seiner Verurteilung freiwillig ausreisen wird. Die Verteidigung wegen der Straftat eines Angeklagten, der einen gesicherten ausländerrechtlichen Status hat, ist somit eine völlig andere als bei denjenigen, die sich in einer ausländerrechtlichen Grauzone befinden.

Dies erklärt zumindest auch, warum gerade in den 1990er Jahren in Deutschland, in der Zeit also, in der die Asylbewerberzahlen so hoch wie nie davor und nie danach waren, so viele Nichtdeutsche als Strafgefangene in Haftanstalten einsaßen. Sind diese „vollziehbar" zur Ausreise verpflichtet, gilt für sie nicht nur wegen des mit dem Strafrecht verschränkten Ausländerrecht ein anderes Recht, sondern insgesamt auch kein positiv spezialpräventives Strafrecht. Alle Instrumente der Haftvermeidung versagen bei dieser Gruppe von Beschuldigten. Stattdessen geht es immer auch um die Abschiebung dieses „ausländischen“ Straftäters. Ignoriert wird sogar die Tatsache, dass viele von ihnen in Deutschland geboren und hier sozialisiert worden sind - mit allen Kulturkonflikten, die das mit sich bringt und den Folgen. Am Beispiel der nichtdeutschen Straftäter kann man also studieren, wie ein im wesentlichen nur noch negativ spezialpräventives Strafrecht wirkt.
II. Wie reflektiert sind die Annahmen der Bundesregierung (Periodischer Sicherheitsbericht) und empirische Rückfallstudien bei der Einschätzung der Straffälligkeit junger Männer des Zuwanderungsmilieus?

Der Zweite Periodische Sicherheitsbericht der Bundesregierung (2006) konstatiert lediglich, dass Dunkelfeldstudien und die Daten der PKS im Hellfeld zu unterschiedlichen Ergebnissen kommen und konstatiert: „Die ganz überwiegende Mehrheit der Ausländer in Deutschland tritt strafrechtlich nicht in Erscheinung" (Kurzfassung 4.2.3 Maßnahmen und Perspektiven der Bundesregierung). Bei der allgemeinen Kriminalitätsbelastung nach der PKS wird konstatiert, dass eine Vielzahl von Delikten nur von Ausländern, insb. solchen mit prekärem ausländerrechtlichen Status begangen werden können. Gleichwohl wird dann aber - wie auch ansonsten im kriminologischen Schrifttum - unterstellt, dass das Verhalten und die Lebenssituation Ursache für die Mehrbelastung im Hellfeld sei, wobei der Grund in Integrationsproblemen gesehen wird. Insgesamt bedeutet dies, dass wir zurzeit noch keine verlässlichen Daten darüber haben, ob nicht die Reaktionen des Rechts und der Rechtsanwender gravierende Unterschiede aufweisen und wo diese liegen.

\section{Gibt es Anhaltspunkte für eine höhere Rückfallgefähr- dung dieser Minderheiten insb. bei der sog. Gewaltkri- minalität?}

Im folgenden soll die Studie von Stefan Harrendorf (Rückfälligkeit und kriminelle Karrieren von Gewalttätern, Göttinger Studien zu den Kriminalwissenschaften Band 12007 ) herangezogen werden, um einzuschätzen, wie belastbar die übliche Annahme ist, dass junge Männer aus dem Zuwanderermilieu insbesondere bei der sog. Gewaltkriminalität überrepräsentiert seien. Denn wenn dies stimmen sollte, dann wäre es etwas plausibler, wenn dementsprechend auch die Reaktionen härter wären. Aber wie so oft erweist sich auch diese Annahme als zu schnell für selbstverständlich gehalten.

Nimmt man die Zahlen der PKS, ergibt sich zunächst einmal ein paradoxer Befund. Vordergründig ist dies nicht der Fall. Die Anteile sind überall etwa gleich hoch, was aber wohl nur damit zusammen hängt, dass eine große Zahl von Delikten außerhalb der hier thematisierten Gewaltkriminalität nur Ausländer begehen können bzw. dass viele Delikte im Zusammenhang mit solchen ausländerrechtlichen Verstößen stehen. Die Belastung dieser Minderheiten bei der sog. Allgemeinen Kriminalität ist also schon deshalb erheblich gesteigert. Sie besagt kriminologisch nichts, es sei denn, man sucht nach Belegen für einen höheren sozialen Druck und tendiert zu einer Anomietheorie.

Wenn aber in allen Deliktsbereichen die Belastung der Nichtdeutschen etwa gleich hoch (etwa 22\% bei einem Anteil an der Wohnbevölkerung von $9 \%$ ) ist, könne man erst aus einem Negativschluss auf eine höhere Belastung der jungen Ausländer bei Gewaltdelikten ohne jeden ausländerrechtlichen Bezug schließen (so auch a.a.O. S. 43). Aber dieser Schluss muss sogleich kontrolliert werden, da ja die Altersstruktur dieser Gruppe eine völlig andere als die der Deutschen ist. Was daher die spezifische Belastung mit Gewaltkriminalität im Hellfeld der polizeilichen Registrierung betrifft, verweist daher auch diese Studie auf die Dunkelfeldforschung. Erst wenn sich dort bestätigen lassen sollte, dass Deutsche und Nichtdeutsche zumindest bei der Frage physischer Gewalt ein unterschiedliches Verhalten zeigen, steigt die Erklärungskraft der Daten im Hellfeld. Aber genau dies ist nicht mehr so unbestritten wie noch vor einigen Jahren.

Die Studie von Stefan Harrendorf geht dieser Frage nicht mehr nach, sondern legt den Schwer- punkt auf die Analyse der Daten des Bundeszentralregisters und beschränkt sich auf die Frage der Rückfälligkeit der wegen eines Gewaltdeliktes Verurteilten. Bezogen auf die uns hier interessierende Frage ist das Ergebnis spannend. Beide Gruppen haben keine unterschiedlichen Rückfallquoten. Zwar gebe es nach der PKS bei Ausländern eine deutlich höhere Häufigkeitsziffer bei sexueller Gewalt, Raub und gefährlicher Körperverletzung, während Tötungsdelikte unauffällig sind (S. 153), nicht aber eine höhere Belastung mit Rückfällen, wenn man die Daten des Bundeszentralregisters heranzieht. Aber der Verfasser macht zugleich deutlich, dass die methodischen Probleme solcher Vergleiche nicht zu übersehen sind. So kommen etwa junge Deutsche häufiger in den Genuss von Einstellungen nach $\S \S 45,47$ JGG als Nichtdeutsche, so dass es wohl unumgänglich ist, den Sanktionsstil genauer zu erforschen. Auch die Häufigkeit von deliktsspezifischen Rückfällen könne im einzelnen über- und unterschätzt werden. Bei den Gewaltdelikten könne sie systematisch unterschätzt werden, da Ausländer wohl häufiger nach einer Verurteilung wegen eines solchen Deliktes abgeschoben werden. Wenn aber bei den Nichtdeutschen eine der Folgen einer Straftat die Abschiebung sein kann, lässt sich nicht genau sagen, ob die Bezugsentscheidungen, auf welche die Rückfälle bezogen werden, nicht $\mathrm{zu}$ sehr von der Abschiebepraxis beeinflusst worden sind (S. 388). Es könnte also sein, dass gerade diejenigen mit der schlechtesten Prognose abgeschoben worden sind, so dass die Ausgangszahl geringer und damit die Rückfälligkeit der nicht Abgeschobenen systematisch unterschätzt wird.

Offenbar haben wir beim Thema Ausländerkriminalität zu lange aus Hellfelddaten Rückschlüsse gezogen, die mit den verzerrten Daten nur bedingt sinnvoll sind. Die spezifische Kriminalitätsbelastung und die spezifischen Reaktionsstile aller Strafverfolgungsbehörden auf Deutsche und Nichtdeutsche 
können erst dann realistisch eingeschätzt werden, wenn diese Verzerrungen untersucht und analysiert worden sind. Zurzeit arbeiten wir mit zu vielen Vermutungen. Es kann sein, dass sich die Annahme einer relativ höheren Belastung der jungen Ausländer bei Delikten wie sexueller Nötigung, Raub und Körperverletzung als zutreffend erweisen, aber noch ist unser Wissen zu lückenhaft, um zeigen zu können, woran es liegt, dass am Ende die Gefangenenquote der jungen Ausländer wesentlich höher ist als bei jungen Deutschen.

Vorrangig sind Studien zur Sanktionspraxis bei jungen Deutschen und Nichtdeutschen, insbesondere die Rolle der ausländerrechtlichen Entscheidungen.

Bei den Gewaltdelikten müsste genau untersucht werden, ob es tatsächlich Unterschiede in der Belastung zwischen Deutschen und Nichtdeutschen gibt. Ferner wie sich bei gerade dieser Deliktsgruppe die ausländerrechtlichen Entscheidungen auswirken; denn dies hat Konsequenzen bei der Frage, ob wir deren Rückfälligkeit systematisch unterschätzen oder nicht. Sollte sich dies als falsch erweisen, dann drängen sich ganz andere Fragen auf. Begünstigt das Ausländerrecht einen Sanktionsstil, der bei allen jungen Ausländern eher negativ spezialpräventive
Entscheidungen nahe legt, oder produziert umgekehrt erst die erhöhte Belastung einzelner Risikogruppen mit Gewaltdelikten unter den Nichtdeutschen eine härtere Gangart? Welche Rolle spielen die Drogendelikte? Schließlich geht hier § 54 Ziff. 3 AufenthaltsG davon aus, dass die Abschiebung der Regelfall sein soll. Es spricht vieles dafür, dass es kaum eine Deliktsgruppe gibt, bei der das Ausländerrecht nicht den Reaktionsstil beeinflusst. Bei typischen Ausländerdelikten versteht sich das von selbst. Desgleichen bei Betäubungsmitteldelikten. Hingegen wirkt das Ausländerrecht bei Taten wie Raub, Vergewaltigung und Körperverletzungen über die erhöhte Straferwartung und die Überlegung, dass dies besonders gefährliche Taten sind. Es scheint so, als verstärke der jederzeit mögliche ausländerrechtliche Zugriff den sozialen Druck erheblich und damit auch auf die hier geborenen Migranten. Erhöhter Druck wirkt ambivalent. Einerseits diszipliniert er, andererseits erschwert er die Integration. Eines ist jedenfalls klar geworden: ohne die Rolle des Ausländerrechts lässt sich kriminologisch wenig sagen über einzelne Risikogruppen unter den hier lebenden Minderheiten und damit zu wenig zum komplexen Thema Strafrecht und Migration.

\section{Polizeilicher Umgang mit Jugendlichen aus dem Zuwanderungsmilieu: \\ Die Reichweite von Interkultureller Kompetenz \\ Reza Ahmari und Joachim Kersten}

Sis eit einiger Zeit geistert der Begriff „Muslimische Jugend $\checkmark$ in Deutschland" durch die Medien und man fragt sich, ob dieser Begriff nicht mehr Kohärenz suggeriert als bei Jugendlichen aus den entsprechenden Herkunftskulturen tatsächlich vorhanden ist. Würden sich deutsche Jugendliche in Nordafrika oder in der Türkei mit der Bezeichnung „Christenjugend“ zutreffend eingeordnet fühlen? Nehmen wir das Beispiel des Rollbergviertels in Berlin-Neukölln, eben das Wohnviertel, aus dem viele Besucher der mittlerweile berüchtigten Rütli-Schule stammen. Die dort lebenden Familien von Türken, Kurden und Libanesen („Araber") haben einen gemeinsamen Status von „nicht - angekommenen“ Zuwanderern. Sozialstrukturell betrachtet unterschichten sie die einheimische Unterschicht noch einmal. Aber zusammenführen oder verbinden tut sie der Islam in keiner Weise. Im Gegenteil, bei interkulturellen Konflikten dienen „religiöse Versatzstücke" der Legitimation von Verhaltensweisen, die mit Religion nichts zu tun haben, sondern „alltägliche gewalttätige Kommunikationsformen" (Heinemann 2006: 111) in den Familien und im Gleichaltrigenbereich legitimieren sollen.

\section{Situationseinschätzung:}

Im folgenden werden nur Stichworte genannt, denn die Tendenz zur Ausgrenzung von Zugewanderten durch die Mehrheitsgesellschaft rührt aus mehreren Faktoren. Einer davon ist das gegenwärtige deutsche Schulsystem, ein anderer ist die „Ihr gehört nicht hierhin!“ Haltung zu vieler Deutscher gegenüber Menschen aus anderen Kulturen. Andererseits löst auf dem Konfliktschauplatz öffentlicher Raum die Sichtbarkeit von Cliquen fast nur männlicher Jugendlicher aus dem Milieu der Migranten bei den Einheimischen Angst und Gefühle der „Überfremdung" aus. Gleichzeitig ist das „Aufmerksamkeit-Zollen“, das die Gruppierungen bei Passanten und Anwohnern erzwingen, für die Jugendlichen/ jungen Männer ein nicht zu unterschätzender Identitätsgewinn. Fast schon „natürlich“ sind solche Cliquen Männerbünde, die die Grenze zwischen Abenteuer und Straftat einigermaßen beharrlich aufsuchen. Die Folge ist deren erhöhte Sichtbarkeit in der Kriminalstatistik, die auch mit der größeren Anzeigebereitschaft der Einheimischen zu tun hat, wenn Straftaten durch „ausländisch“ wirkende Jugendliche begangen werden.

Im Konfliktzentrum Familie wirkt sich außerdem die Kontrolle der Mädchen negativ aus: Sie dür- fen nicht mehr ins Jugendzentrum und von ihnen wird die Durchsetzung traditioneller Rollenskripts verlangt. Dazu gesellen sich Konflikte ökonomischer Natur sowie Grundrechtsverletzungen, die aufgrund von traditionell-patriarchalischen Normen erfolgen. Kombiniert mit einem fundamentalistischen Verständnis von Religion findet häufig Gewaltausübung durch Väter/ Brüder statt. Angesichts solcher Konflikte sind die Grenzen des Konflikt regulierenden Instrumentariums der Jugend- und Sozialarbeit schnell erreicht. Hier ist eine Kooperation mit anderen sozialen Diensten, auch mit Vertretern der Polizei unabdingbar. Das Ziel muss sein, Netzwerke zu schaffen, in denen sich interkulturelle und professionelle Kompetenz bündeln lässt.

Praktiker der Jugendarbeit und -hilfe beobachten, dass bei Konflikte unter Jugendlichen aus dem 